\title{
Árvores fixadoras de nitrogênio e macrofauna do solo em pastagem de híbrido de Digitaria
}

\author{
Paulo Francisco Dias(1), Sebastião Manhães Souto(2), Maria Elizabeth Fernandes Correia(2), \\ Gudesteu Porto Rocha ${ }^{(3)}$, Joventino Fernandes Moreira( ${ }^{(2)}$, Khalil de Menezes Rodrigues ${ }^{(2)}$ e Avílio Antônio Franco ${ }^{(2)}$ \\ (1)Pesagro, BR 465, Km 7, CEP 23890-000 Seropédica, RJ. E-mail: pfranciscodias@hotmail.com.br (2)Embrapa Agrobiologia, BR 465, Km 7, \\ CEP 23851-970 Seropédica, RJ. E-mail: smsouto@cnpab.embrapa.br, ecorreia@cnpab.embrapa.br, jovfmrural@yahoo.com.br, \\ agrokhalil@yahoo.com.br, avilio@cnpab.embrapa.br(3)Universidade Federal de Lavras, Dep. de Zootecnia, Caixa Postal 3.037, \\ CEP 37200-000 Lavras, MG. E-mail: gudesteu@ufla.br
}

\begin{abstract}
Resumo - O objetivo deste trabalho foi avaliar o efeito de três leguminosas arbóreas sobre a densidade e a diversidade de macrofauna de um Argissolo Vermelho-Amarelo, de baixa fertilidade natural. Duas espécies fixadoras de nitrogênio atmosférico, a orelha-de-negro (Enterolobium contortisiliquum) e o jacarandá-da-baía (Dalbergia nigra), e uma não-fixadora, o angico-canjiquinha (Peltophorum dubium), foram consorciadas em pastagem de capim survenola (híbrido interespecífico entre Digitaria setivalva e Digitaria valida), tendo por testemunha pasto a pleno sol. Formicidae foi o grupo mais abundante em todos os tratamentos, sendo seguido por Oligochaeta, com $47 \%$ nos tratamentos com leguminosas e $23 \%$ no pasto a pleno sol. Os maiores valores em diversidade de fauna foram obtidos nas amostragens sob as copas das leguminosas fixadoras de $\mathrm{N}_{2}$. A análise multivariada de agrupamento mostrou que o consórcio formado com orelha-de-negro apresentou grupos de fauna bastante semelhantes ao do consórcio formado com jacarandá-da-baía. De acordo com a análise multivariada de correspondência, as leguminosas arbóreas contribuíram para aumentar a densidade de alguns grupos de fauna, principalmente Oligochaeta, Coleoptera, Araneae e Formicidae.
\end{abstract}

Termos para indexação: sistema silvipastoril, leguminosas arbóreas, fauna do solo.

\section{Nitrogen-fixing trees and soil macrofauna in Digitaria hybrid pasture}

\begin{abstract}
The objective of this work was to evaluate the effect of tree legume species on the density and diversity of macrofauna of a Red Yellow Argisol with low natural fertility. Two nitrogen-fixing trees, orelha-denegro (Enterolobium contortisiliquum) and jacarandá-da-baía (Dalbergia nigra), and one non nitrogen-fixing tree, angico-canjiquinha (Peltophorum dubium), were intercropped with survenola grass (an interspecific hybrid of Digitaria setivalva and Digitaria valida), having pasture at full sun as control. Formicidae was the most abundant group, followed by Oligochaeta with $47 \%$ in the legume treatments and $23 \%$ in the pasture at full sun. The largest values for the fauna diversity were obtained in samplings under the canopy of nitrogen-fixing tree legumes. The cluster analysis showed that the intercropping with orelha-de-negro presented soil fauna groups quite similar to the ones under jacarandá-da-baía. According to the correspondence multivariate analysis, the tree legumes contributed to an increase in the density of some fauna groups, mainly Oligochaeta, Coleoptera, Araneae and Formicidae.
\end{abstract}

Index terms: silvipastural system, tree legumes, soil fauna.

\section{Introdução}

O avanço da agricultura de monocultivo e da pecuária, em busca de novas áreas para plantio e formação de pastagens, em várias regiões do Brasil e do mundo, é uma das principais fontes de degradação dos recursos naturais, em virtude da utilização de técnicas agrícolas inadequadas e incompatíveis com o desenvolvimento sustentável. Na maior parte do Brasil, após o esgotamento do solo com culturas anuais, pastagens têm sido estabelecidas. Na Amazônia e em algumas partes do Cerrado, as pastagens estão sendo formadas em áreas de floresta, utilizando-se queimadas, seguidas de plantio de culturas anuais, com semeadura de forrageiras no segundo ano.

A minimização dos impactos ambientais da pecuária, por meio da arborização de pastagens, em particular com 
leguminosas arbóreas fixadoras de $\mathrm{N}_{2}$, é uma alternativa que visa a incrementar a qualidade do solo.

Nos sistemas silvipastoris, os principais aportes ao subsistema decompositor são o material foliar e o sistema radicular, que irão constituir a serapilheira. $\mathrm{Na}$ serapilheira e nos primeiros centímetros do solo, ocorre uma intensa decomposição e ciclagem de nutrientes, e são os invertebrados saprófagos, que aí vivem, os principais agentes dessas transformações.

O aumento do número de indivíduos de espécies da macrofauna do solo ocorre pela disponibilidade de melhores condições ambientais, que favorecem a reprodução dos invertebrados, conforme relatado por Seeber et al. (2005), e que se revelam por meio do índice de diversidade e do índice de uniformidade (Brown et al., 2004).

Observações de Walker (1998) mostram que a diversidade da fauna do solo, expressa pelo índice de diversidade de Shannon, está associada a uma relação entre a riqueza (número de grupos) e a uniformidade (distribuição do número de indivíduos entre os grupos); para Odum (1983), o índice de Shannon tem-se mostrado útil em comparações de fauna de solo, entre tratamentos.

O objetivo deste trabalho foi avaliar o efeito de três espécies arbóreas - duas leguminosas fixadoras de $\mathrm{N}_{2}$ e uma não fixadora -, na densidade e diversidade da macrofauna invertebrada do solo, avaliando-se também a predominância de grupos de macrofauna, em conformidade com a cobertura vegetal.

\section{Material e Métodos}

O estudo foi conduzido no campo experimental pertencente ao Sistema Integrado de Produção Agroecológico (SIPA) - Fazendinha do Km 47, Seropédica, RJ, em uma pastagem formada há dez anos, em um Argissolo Vermelho-Amarelo de baixa fertilidade natural, com capim survenola, um híbrido interespecífico de Digitaria setivalva x D. valida.

As espécies arbóreas utilizadas foram duas leguminosas fixadoras de $\mathrm{N}_{2}$, o jacarandá-da-baía (Dalbergia nigra) e a orelha-de-negro (Enterolobium contortisiliquum) e uma não fixadora, o angicocanjiquinha (Peltophorum dubium). Os dados dendrométricos das espécies arbóreas, na área de pastagem no SIPA, depois de dez anos da implantação, foram 7,6 e 6,6 m, 10,8 e 8,3 m, e 3,6 e 3,8 m, para o raio da copa e altura da planta do jacarandá-da-baía, orelha- de-negro e angico-canjiquinha, respectivamente. $\mathrm{Na}$ adubação de plantio das leguminosas, foram aplicados $200 \mathrm{~g}$ de uma mistura de 20 partes de cinza +10 partes de termofosfato +5 partes de calcário $+10 \mathrm{~g}$ de FTE BR-12, em covas de 20x20x20 cm, com espaçamento de $15 \times 15 \mathrm{~m}$ entre plantas. O plantio no campo foi de mudas com inoculação de estirpes eficientes de rizóbio (BR 8401 + BR 8409, para jacarandáda-baía; BR 4406 + BR 4407, para orelha-de-negro; BR 3617 + BR 3609, para angico-canjiquinha) e fungos micorrízicos (Gigaspora margarita e Glomus macrocarpum) da coleção da Embrapa Agrobiologia. As mudas foram transplantadas para o campo em março de 1994.

Antes do experimento, no período chuvoso de 2003, a pastagem vinha sendo mantida sob pastejo rotativo, com período de descanso de 45 a 60 dias no período da seca, e de 30 a 42 dias no período das chuvas.

$O$ delineamento experimental utilizado foi o inteiramente casualizado, em parcela subdividida, com três repetições, onde as árvores, em um total de três por espécie, mais um tratamento só com capim, representaram as parcelas, e as amostragens representaram as subparcelas.

Os efeitos das leguminosas arbóreas sobre a macrofauna do solo foram avaliados por meio de amostragens feitas a $30 \mathrm{~cm}$ de profundidade, na metade da distância do raio de projeção da copa e fora da influência da copa da árvore. Foram utilizados dois pontos de amostragem por planta, na direção norte-sul, num total de seis amostras. Foi amostrado o solo sob a copa de jacarandá-da-baía, de orelha-de-negro e de angicocanjiquinha, e na área com o capim survenola. As amostragens foram feitas em novembro de 2003, no início do período chuvoso.

O método utilizado para retirada das amostras foi o recomendado pelo programa Tropical Soil Biology and Fertility (TSBF) para estudar a macrofauna inserida nas camadas do solo, descrito por Anderson \& Ingram (1993). O método consistiu em delimitar a área amostral com auxílio de um quadrado metálico de $25 \times 25 \mathrm{~cm}$. Abriu-se uma trincheira de $30 \mathrm{~cm}$ de profundidade para facilitar a retirada da terra a ser analisada. A terra foi acondicionada em sacos de plástico e, em seguida, procedeu-se à separação da macrofauna contida na terra e na serapilheira, utilizando-se uma bandeja, de onde a fauna, vista a olho nu (macrofauna), foi coletada e acondicionada em frascos identificados, contendo álcool 70\% para fixação. 
No laboratório, o conteúdo dos recipientes foi examinado sob lupa binocular. Os indivíduos de cada frasco foram contados e identificados em grandes grupos taxonômicos, em geral ordens, de acordo com as descrições fornecidas por Dindal (1990).

Os dados relativos ao número de indivíduos por metro quadrado e o respectivo erro-padrão da média foram obtidos a partir da média dos grupos, em cada tratamento formado com o capim survenola. O índice de diversidade de Shannon foi estimado a partir da fórmula: $\mathrm{H}=-\Sigma$ pi. $\log _{2}$ pi, em que pi é a proporção de indivíduos i, coletados na amostragem (Magurran, 1988). A fórmula para calcular a uniformidade de Pielou foi derivada a partir de $\mathrm{H}$, ou seja, $\mathrm{U}=\mathrm{H} / \log _{2} \mathrm{R}$, em que $\mathrm{R}$ é a riqueza definida como número de grupos taxonômicos, encontrados em cada tratamento. A riqueza média (RM) representa o número médio de grupos da fauna coletados por amostra, em cada tratamento.

Foi feita uma avaliação das dissimilaridades entre as comunidades de macrofaunas do solo, por meio de análise multivariada de agrupamentos, com o complemento do coeficiente de correlação de Pearson como medida de distância, usando-se, ainda, o método de ligação completa, segundo Ribeiro Junior (2001), com o objetivo de identificar agrupamento de tratamentos com maior ou menor grau de similaridade. A riqueza média (RM) representa o número médio de grupos da fauna coletados por amostra, em cada tratamento.

A análise de correspondência ou análise fatorial de correspondência, usada com um método de ordenamento dos tratamentos (leguminosas ou só pasto) e das variáveis (grupos de fauna do solo), foi feita seguindose a metodologia de Lepš \& Šmilauer (2003), a fim de identificar os grupos de fauna mais correlacionados, positiva ou negativamente, com os tratamentos.

\section{Resultados e Discussão}

Observou-se maior densidade de fauna no solo sob influência da leguminosa angico-canjiquinha, seguido pela pastagem sem árvore, pela leguminosa orelha-de-negro e pelo consórcio formado com o jacarandá-da-baía (Tabela 1).

A maior variabilidade da densidade na macrofauna do solo, entre os consórcios formados, ocorreu entre as áreas de projeção da copa de angico, enquanto que os valores das amostras coletadas nas áreas de projeção da copa, da espécie fixadora orelha-de-negro, e área de pasto sem árvore foram menores e ficaram próximas (Tabela 1).

Os maiores valores de $\mathrm{H}$ foram obtidos com as amostragens realizadas sob as copas de orelha-de-negro e jacarandá-da-baía, seguidos pelos índices obtidos sob influência da copa de angico-canjiquinha e, por último, na área de pasto a pleno sol (Tabela 1). Portanto, a presença de espécies arbóreas na pastagem favoreceu a diversidade da fauna de solo, em relação à pastagem sem a presença de leguminosa.

A presença de uma leguminosa arbórea cria condições favoráveis à fauna, pela deposição da serapilheira, diminuindo a relação $\mathrm{C} / \mathrm{N}$ sob a sua copa, além de estabelecer um microclima mais favorável. Conseqüentemente, é esperado um aumento do número de espécies e do número de indivíduos por espécie, em decorrência da disponibilidade de fonte de energia e $\mathrm{N}$, que favorecem a reprodução dos invertebrados, conforme relatado por Joffre et al. (1988) e Hang et al. (1995), e que se revelam por meio do $\mathrm{H}$ e do índice de uniformidade (Tabela 1).

Os maiores valores de riqueza total, encontrados para as leguminosas, em comparação com o pasto a pleno sol (Tabela 1), podem ser explicados pelo microclima e pela serapilheira oriunda das leguminosas arbóreas, conforme as informações registradas por Frank \& Furtado (2001), já que os outros fatores, tais como solo, tipo de manejo e pastagem se mantiveram os mesmos.

Apesar de dados meteorológicos serem os mesmos, para todas as espécies, não se pode inferir qual a relação entre os diferentes fatores do clima influiu na riqueza sob os diferentes tipos de copa. Segundo relato de Frank \& Furtado (2001), no Estado do Acre, tanto no período seco quanto no chuvoso, observou-se um

Tabela 1. Densidade, em número de indivíduos por metro quadrado, com respectivos erros-padrões, diversidade, riqueza total, riqueza média e uniformidade da fauna de solo na camada de $0-30 \mathrm{~cm}$, sob pastagem de capim survenola, consorciado com três leguminosas.

\begin{tabular}{llcccc}
\hline Consórcio & Densidade & Diversidade & Riqueza total & Riqueza média & Uniformidade \\
\hline Orelha-de-negro & $1.930 \pm 561$ & 1,10 & 13 & 6 & 0,30 \\
Jacarandá-da-baía & $1.554 \pm 253$ & 1,05 & 13 & 7 & 0,28 \\
Angico-canjiquinha & $3.317 \pm 1726$ & 0,81 & 18 & 6 & 0,19 \\
Pasto & $2.213 \pm 867$ & 0,62 & 8 & 6 & 0,21 \\
\hline
\end{tabular}


aumento significativo da macrofauna no solo sob a copa das árvores, fator esse que foi relacionado às condições do microclima.

Os maiores valores de uniformidade foram gerados nas amostragens realizadas na área de pastagem sob a copa de orelha-de-negro $(0,30)$ e jacarandá-dabaía $(0,28)$ (Tabela 1). A partir dessa relação, pôde-se estabelecer que as amostras coletadas sob a copa dessas espécies apresentaram menor dominância de grupos do que na área de pasto e do que na área de pasto sob influência da copa de angico-canjiquinha. Essas condições podem sugerir que o microclima e os recursos alimentares foram capazes de atrair mais indivíduos de diferentes grupos, funcionando como uma espécie de refúgio na pastagem.

De acordo com a relação feita por Begon et al. (1996) para uniformidade, quanto menores forem os valores obtidos para esse índice, menos uniforme é o ecossistema estudado; assim, os grupos ou um grupo específico de organismos pertencentes a esta comunidade possuiriam uma dominância mais acentuada que outros.

As espécies orelha-de-negro e jacarandá-da-baía apresentaram uma riqueza intermediária de grupos, com diversidade e uniformidade maiores; o pasto sem árvores apresentou menor riqueza de grupos; e a área de pasto sob influência da copa de angico-canjiquinha proporcionou condições para maior riqueza de grupos, ambas com menor diversidade, fazendo com que a uniformidade da fauna em suas áreas fosse menor. Desta forma, foi possível concluir que os consórcios existentes atuaram como reguladores das densidades de macrofauna, já que o solo foi o mesmo para as quatro condições.
O grupo Formicidae se destacou nos consórcios formados pelas leguminosas arbóreas e a pastagem de capim survenola (Tabela 2). A maior representatividade deste grupo, acima de $80 \%$ do total de indivíduos, em cada consórcio, está associada à topografia que favorece o escoamento da água, pois, segundo Santos (2001), as formigas preferem construir seus formigueiros em terrenos que não acumulam água.

Quando desconsiderado o grupo Formicidae, o segundo grupo mais expressivo foi o Oligochaeta na presença das leguminosas, em relação ao pasto, o que indica a forte contribuição das leguminosas na densidade desse grupo (Tabela 2). Entre as leguminosas, esperava-se que a orelha-de-negro, comparada ao angico-canjiquinha, por apresentar menor relação C/N (Silva et al., 2004), apresentasse maior densidade de oligoquetas. Entretanto, os resultados encontrados discordam dos de Froufe (1999) que, ao trabalhar com Eucalyptus grandis, Pseudosamanea guachapele e Acacia mangium, encontrou maior taxa de decomposição em folhas de leguminosas arbóreas com menor relação $\mathrm{C} / \mathrm{N}$.

Foram encontradas diferenças para a densidade de Oligochaeta, entre a orelha-de-negro e angicocanjiquinha, a favor dessa última espécie, quando desconsiderado o grupo Formicidae (Tabela 2). A população de oligoquetas praticamente dobrou, em todos os consórcios, quando comparada às amostragens realizadas na área de pasto. Esses resultados vêm ao encontro dos obtidos por Frank \& Furtado (2001) e, também, das experimentações feitas por Wild et al. (1993), em três locais de Queensland, Austrália, quando trabalharam com restrições de luz em gramíneas, e verifica-

Tabela 2. Distribuição relativa (\%) dos grupos funcionais da fauna de solo, em consórcios do capim survenola com leguminosas arbóreas e só no pasto, em análises realizadas com inclusão e sem inclusão do grupo Formicidae.

\begin{tabular}{|c|c|c|c|c|}
\hline Grupo funcional & Orelha-de-negro & Jacarandá-da-baía & Angico-canjiquinha & Pasto \\
\hline \multicolumn{5}{|c|}{ Com o grupo Formicidae } \\
\hline Formicidae & 82,6 & 83,5 & 88,4 & 91,4 \\
\hline Larvas de Coleoptera & 2,5 & 1,9 & 0,3 & 2,7 \\
\hline Oligochaeta & 7,0 & 8,2 & 5,7 & 1,9 \\
\hline Araneae & 1,5 & 1,5 & 0,9 & 1,2 \\
\hline Isopoda & 0,0 & 0,7 & 2,3 & 0,0 \\
\hline Coleoptera & 2,5 & 2,4 & 0,5 & 2,2 \\
\hline Outros & 3,8 & 1,7 & 1,9 & 0,6 \\
\hline \multicolumn{5}{|c|}{ Sem o grupo Formicidae } \\
\hline Larvas de Coleoptera & 14,3 & 11,5 & 2,8 & 31,0 \\
\hline Oligochaeta & 40,6 & 50,0 & 49,3 & 22,5 \\
\hline Araneae & 8,7 & 9,4 & 7,6 & 14,1 \\
\hline Isopoda & 0,0 & 4,2 & 19,4 & 0,0 \\
\hline Coleoptera & 14,3 & 14,6 & 4,2 & 25,4 \\
\hline Outros & 22,1 & 10,4 & 16,7 & 7,0 \\
\hline
\end{tabular}


ram um aumento no número de minhocas em solos coletados em parcelas de gramíneas submetidas a 50\% de restrição de luz, fator esse relacionado às condições microambientais provocadas pela sombra, embora não houvesse contribuição da serapilheira.

A fim de se evidenciarem dissimilaridades entre as comunidades da macrofauna do solo, aplicou-se a análise multivariada de agrupamento, com o complemento do coeficiente de correlação de Pearson como medida de distância e com o método de ligação completa. A partir dessa análise, foram obtidos dendrogramas, nos quais foram identificados agrupamentos de tratamentos com maior ou menor grau de similaridade, de acordo com sua distância.

O método de ligação completa, também chamado de método de aglomeração por diâmetro ou, ainda, de vizinho mais distante, caracteriza-se pela formação de grupos a partir da fusão dos dois elementos mais distantes, e é o método mais recomendado em ecologia, quando o objetivo é identificar fortes descontinuidades (Valentin, 1995).

A análise de agrupamento identificou dois conjuntos de tratamentos, o formado pelas três leguminosas e o do pasto sem árvores, que não apresentaram nenhuma similaridade entre si, uma vez que sua distância de ligação foi de $100 \%$ (Figura 1).

O consórcio formado com jacarandá-da-baía e orelhade-negro apresentou ocorrência semelhante de grupos da fauna, tendo divergido 15\% entre si. Na comparação das ocorrências desse grupo com angico-canjiquinha foi

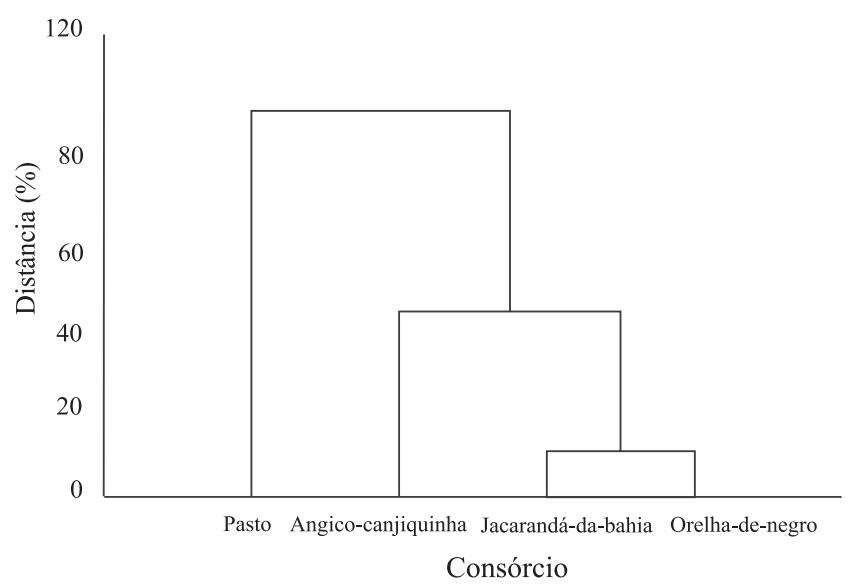

Figura 1. Dendrograma da densidade dos diferentes grupos, resultante dos tratamentos considerados, por meio do complemento do coeficiente de correlação de Pearson, como medida de distância, e com o método de ligação completa. apontada, por sua vez, divergência de 50\% das distâncias calculadas. Quanto à densidade, no consórcio formado com angico-canjiquinha, observou-se grande diferenciação em relação aos outros tratamentos. Isto significa que, no conjunto geral dos consórcios estudados, o formado com angico-canjiquinha apresentou um padrão diferenciado do que ocorreu com o pasto puro e com as outras duas leguminosas.

O angico-canjiquinha é uma leguminosa não fixadora de $\mathrm{N}_{2}$. A forte presença do grupo Formicidae, nesse consórcio, pode ser justificada pela baixa contribuição desta árvore com serapilheira com alta concentracão de N, portanto, com baixa relação C/N (Silva et al., 2004).

A análise de correspondência, também conhecida como análise fatorial de correspondência, foi utilizada como método de ordenamento dos pontos tratamentos formados pelos consórcios de leguminosas arbóreas e o pasto em monocultivo, e pelos pontos variáveis formados pelos grupos da fauna do solo.

O diagrama de ordenação dos pontos variáveis e dos pontos tratamentos formados foi montado apenas com os dois primeiros eixos ou fatores extraídos, uma vez que estes totalizaram $88 \%$ da variância existente (Figura 2).

A proximidade dos dois consórcios pontos tratamentos e os pontos variáveis, no plano, traduzem maior correlação entre esses tratamentos, possivelmente por se tratarem de leguminosas fixadoras de $\mathrm{N}_{2}$, e por estarem distantes, positivamente, do centro de projeção do eixo II. O ponto variável formado por larva de Diptera, Gastropoda e Isopoda, próximo ao ponto tratamento angico-canjiquinha de forma positiva, significa que, em média, esses grupos têm alto valor correlacionado nesse ponto.

No ponto tratamento pasto, observou-se um afastamento dos eixos de forma negativa, em relação aos outros pontos tratamentos e também dos pontos variáveis.

O eixo ou fator I, que responde por cerca de $54 \%$ da variância, está positivamente ligado ao ponto tratamento formado por angico-canjiquinha e aos pontos variáveis formados por larvas de Diptera, Isopoda, Gastropoda, Formicidae, Heteroptera, Diplopoda, Oligochaeta, larvas de Formicidae, Dermaptera e Homoptera, o que indica que suas densidades covariam. Além disso, os grupos ocorreram em maior abundância nas amostras das leguminosas, em relação ao pasto a pleno sol. 
Em oposição, os pontos tratamentos formados com as leguminosas fixadoras de $\mathrm{N}_{2}$ projetam-se negativamente no eixo I, com os pontos variáveis formados por Araneae, Coleoptera, larvas de Coleoptera, Blattodea e ovos de Oligochaeta (Figura 2).

Os pontos variáveis formados por Araneae, Heteroptera e Formicidae contribuíram pouco para formação dos dois eixos, sendo que Formicidae tendeu mais para a formação do pasto e para a leguminosa não fixadora de $\mathrm{N}_{2}$.

O fator II totalizou 34\% da variância explicada e esteve positivamente ligado à maioria dos pontos variáveis, exceto para o formado com Formicidae, Isopoda e larvas de Diptera, o que indica que a maior abundância foi observada nos pontos tratamentos formados com orelha-de-negro e jacarandá-da-baía, o que indica maior correlação entre esses tratamentos.

Em contrapartida, pontos variáveis formados por Formicidae e Isopoda estiveram projetados negativamente neste eixo, mas com a sua proximidade do centro do diagrama, pode-se dizer que apresentou uma baixa correlação com o eixo.

A presença da leguminosa na pastagem favorece o aumento dos grupos de invertebrados da fauna de solo. Estudos para a identificação das espécies arbóreas que mais contribuem para esse aumento são necessários, bem como, para avaliar a influência do microclima na macrofauna, sob e fora da copa das árvores. O efeito alelopático também deve ser analisado, uma vez que espécies com características desejáveis para a ciclagem

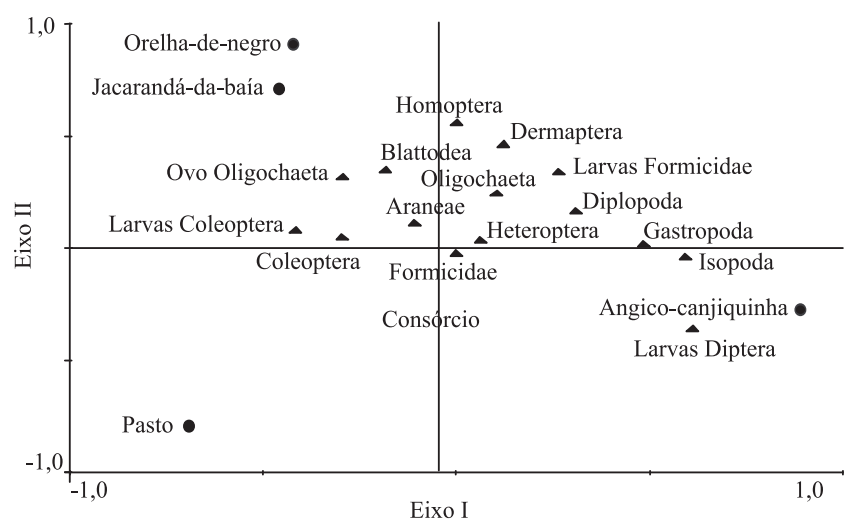

Figura 2. Diagrama de ordenamento, obtido a partir da análise fatorial de correspondência, para densidades médias dos grupos funcionais em uma coleta. Eixo I: 54\% da variância explicada; Eixo II: 34\% da variância explicada; $\Delta$ : pontos variáveis de grupos da fauna explicada; • : pontos tratamentos. de $\mathrm{N}$, no sistema, não proporcionaram o aumento do número de indivíduos de Oligochaeta, considerado como um dos indicadores de qualidade de solo, por decompor biomassa com baixa relação $\mathrm{C} / \mathrm{N}$.

\section{Conclusões}

1. A presença das leguminosas, fixadoras e não fixadoras de $\mathrm{N}_{2}$, contribui para o aumento da densidade, da riqueza e da diversidade da fauna de solo, principalmente dos grupos Oligochaeta, Coleoptera, Araneae e Formicidae.

2. A leguminosa arbórea angico-canjiquinha e o pasto de Digitaria proporcionam melhores condições para a dominância do grupo Formicidae.

3. As leguminosas arbóreas fixadoras de $\mathrm{N}_{2}$ apresentam, sob sua copa, uma fauna do solo mais diversificada.

\section{Referências}

ANDERSON, J.M.; INGRAM, J.S.I. Tropical soil biology and fertility: a handbook of methods. $2^{\text {nd }}$ ed. Wallingford, UK: CAB International, 1993. 221p.

BEGON, M.; HAPER, J.L.; TOWNSED, C.R. Ecology: individuals, populations and communities. $3^{\text {rd }}$ ed. Oxford: Blackwell Science, 1996. 1068p.

BROWN, G.G.; MORENO, A.G.; BAROIS, I.; FRAGOSO, C.; ROJAS, P.; HERNÁNDEZ, B.; PATRÓN, J.C. Soil macrofauna in SE Mexican pastures and the effect of conversion from native to introduced pastures. Agriculture, Ecosystems and Environment, v.103, p.313-327, 2004.

DINDAL, D.L. Soil biology guide. New York: Wiley, 1990. 1348p.

FRANKE, I.L.; FURTADO, S.C. Sistemas silvipastoris: fundamentos e aplicabilidade. Rio Branco: Embrapa Acre, 2001. 51p. (Documentos, 74).

FROUFE, L.C.M. Decomposição de serapilheira e aporte de nutrientes em plantios puros e consorciados de Eucalipytus grandis Maidem, Pseudosamanea guachapele Dungand e Acacia mangium Wild. 1999. 73p. Dissertação (Mestrado) - Universidade Federal Rural do Rio de Janeiro, Seropédica.

HANG, S.; MAZZARINO, M.J.; NUÑES, G.; OLIVA, L. Influencia del desmonte selectivo sobre la disponibilidad de nitrógeno en años húmedos y secos en sistemas silvopastoriles en el chaco árido argentino. Agroflorestería en las Américas, v.2, p.9-14, 1995.

JOFFRE, R.; VACHER, J.; LLANOS, C. de los; LONG, G. The dehesa: an agrosilvopastoral system of the Mediterranean region with special reference to the Sierra Morena area of Spain. Agroforestry Systems, v.6, p.71-96, 1988.

LEPŠ, J.; ŠMILAUER, P. Multivariate analysis of ecological data using Canoco. Cambridge: Cambridge University Press, 2003. 282p. 
MAGURRAM, A.E. Ecological diversity and its measurement. Princeton: Princeton University Press, 1988. 179p.

ODUM, E.P. Ecologia. Rio de Janeiro: Guanabara, 1983. 434p. RIBEIRO JUNIOR, J.I. Análises estatísticas no SAEG. Viçosa: UFV, 2001. 301p.

SANTOS, E.M.R. dos. Densidade, diversidade e biomassa da fauna do solo em serapilheira manipulada numa floresta secundária na Amazônia Central. 2001. 95p. Dissertação (Mestrado) - Instituto Nacional de Pesquisas da Amazônia, Manaus. SEEBER, J.; SEEBER, G.U.H.; KÖSSLER, W.; LANGEL, R.; SCHEU, S.; MEYER, E. Abundance and trophic structure of macrodecomposers on alpine pastureland (Central Alps, Tyrol): effects of abandonment of pasturing. Pedobiologia, v.49, p.221-228, 2005.

SILVA, G.T.A.; QUEIROZ, R.O.M.; NÓBREGA, P.O.; CAMPELLO, E.F.C.; RESENDE, A.S. Caracterização dos teores de nitrogênio, polifenol e relação C:N no tecido foliar de diferentes espécies vegetais em um sistema silvipastoril. In: JORNADA CIENTÍFICA DA UNIVERSIDADE FEDERAL RURAL DO RIO DE JANEIRO, 14., 2004, Seropédica. Anais. Seropédica: Ed. da Universidade Rural, 2004. p.55-59.

VALENTIN, J.L. Agrupamento e ordenação. In: PERES-NETO, P.R.; VALENTIN, J.L.; FERNANDEZ, F.A.S. (Ed.). Oecologia Brasiliensis: tópicos em tratamento de dados biológicos. Rio de Janeiro: Programa de Pós-Graduação em Ecologia, Instituto de Biologia, UFRJ, 1995. p.27-55.

WALKER, D. Diversity and stability. In: CHERRET, J.M. (Ed.). Ecological concepts. Oxford: Blackwell Scientific Publ., 1998. p.115146.

WILD, D.W.M.; WILSON, J.R.; STÜR, W.W.; SHELTON, H.M. Shading increases yield of nitrogen-limited tropical grasses. In: INTERNATIONAL GRASSLAND CONGRESS, 17., Rockhampton. Proceedings. Rockhampton, 1993. p.2060-2062.

Recebido em 28 de agosto de 2005 e aprovado em 10 de janeiro de 2006 